\title{
Point Rupture Solutions of a Class of Quasi-linear Elliptic Equations
}

\author{
Attou A. Miloua \\ Department of Mathematics, CalU, California, PA, 15419 \\ Email: atmpitt@gmail.com
}

\begin{abstract}
Let $\Omega$ be a region in $\mathbb{R}^{2}$ and $f$ be a positive $C^{1}$ function satisfying

$$
\lim _{u \rightarrow 0^{+}} f(u)=\infty .
$$

We consider the quasi-linear elliptic equations of the form

$$
\operatorname{div}(a(u) \nabla u)=\frac{a^{\prime}(u)}{2}|\nabla u|^{2}+f(u)
$$

where $a$ is a positive $C^{1}$ function. Motivated by the thin film equations, a solution $u$ is said to be a point rupture solution if for some $p \in \Omega, u(p)=0$ and $u(p)>0$ in $\Omega \backslash\{p\}$. Our main result is a sufficient condition on $a$ and $f$ for the existence of radial point rupture solutions.
\end{abstract}

Keywords: Thin film, point rupture solution, radial solution, singular elliptic equation, quasilinear elliptic equation.

\section{Introduction}

Let $\Omega$ be a region in $\mathbb{R}^{2}$, and $f$ be a smooth function defined on $(0, \infty)$ satisfying

$$
\lim _{s \rightarrow 0^{+}} f(s)=\infty
$$

we consider the quasi-linear elliptic equations of the form

$$
\operatorname{div}(a(u) \nabla u)=\frac{a^{\prime}(u)}{2}|\nabla u|^{2}+f(u)
$$

where the terms depending upon $a$ are formally associated with the functional

$$
\int_{\Omega} a(u)|\nabla u|^{2}
$$

which can be viewed as a minimizing problem in presence of a Riemannian metric tensor depending upon the unknown $u$ itself.

Motivated by the studies of thin film equations, a solution to (1.2) is said to be a point rupture solution if for some $p \in \Omega, u(p)=0$ and $u(x)>0$ for any $x \in \Omega \backslash\{p\}$. Our main result is the existence of a radial rupture solution:

Theorem 1. Assume that for some $\sigma^{*}>0, a \in C^{1}\left[0, \sigma^{*}\right], f \in C^{1}\left(0, \sigma^{*}\right]$ are positive functions such that for some positive constants $m<M$,

$$
m \leq a(u) \leq M
$$

holds for any $u \in\left[0, \sigma^{*}\right]$ and $f$ is monotone decreasing function on $\left(0, \sigma^{*}\right]$ satisfying

$$
\frac{u}{G(u) f(u)} \in L^{1}\left[0, \sigma^{*}\right]
$$

where

$$
G(u)=\int_{0}^{u} \frac{1}{f(s)} \mathrm{d} s .
$$


Then there exists $r^{*}>0$ and a radial point rupture solution $u_{0}$ to (1.2) in $B_{r^{*}}(0)$ such that $u_{0}=u_{0}(r)$ is continuous on $\left[0, r^{*}\right]$,

$$
u_{0}(0)=0, u_{0}(r)>0 \text { for any } r \in\left(0, r^{*}\right] .
$$

Moreover, $u_{0} \in H^{1}\left(B_{r^{*}}(0)\right)$ and $u_{0}$ is a weak solution to (1.2) in the sense that for any $\varphi \in C_{0}^{\infty}\left(B_{1}\left(r^{*}\right)\right)$,

$$
\int_{B_{1}\left(r^{*}\right)} a\left(u_{0}\right) \nabla u_{0} \nabla \varphi+\frac{a^{\prime}\left(u_{0}\right)}{2}\left|\nabla u_{0}\right|^{2} \varphi+f\left(u_{0}\right) \varphi=0 .
$$

When $a \equiv 1,(1.2)$ is reduced to the simpler form

$$
\Delta u=f(u)
$$

and its rupture solution has been investigated in [4], [6] when $f(u)=u^{-\alpha}-1, \alpha>1$ which has application to the van der Waals force driven thin films, in [5] with $f$ satisfying the growth condition (1.3) and in $[2]$ when the space dimension $\geq 3$. We also remark here that the uniqueness result for general functions $a$ and $f$ is still open. (1.2) has also been studied by F. Gladiali and M. Squassina [1] where they are interested in the so called explosive solutions.

\section{Proof of the Main Result}

We consider the quasi-linear equations of the form

$$
\operatorname{div}(a(u) \nabla u)=\frac{a^{\prime}(u)}{2}|\nabla u|^{2}+f(u)
$$

in a region $\Omega \subset \mathbb{R}^{2}$ where for some $\delta^{*}>0, a \in C^{1}\left[0, \delta^{*}\right]$ and $f \in C^{1}\left(0, \delta^{*}\right]$ are positive functions such that for some positive constants $m<M$,

$$
m \leq a(u) \leq M \text { holds for any } u \in\left[0, \delta^{*}\right] .
$$

Let $g$ be the unique solution to the Cauchy problem

$$
g^{\prime}=\frac{1}{\sqrt{a(g)}}, g(0)=0,
$$

and let $v$ be a solution to

$$
\Delta v=h(v)
$$

where

$$
h(v)=\frac{f(g(v))}{\sqrt{a(g(v))}} .
$$

Define

$$
u=g(v) .
$$

We have

$$
\nabla u=g^{\prime}(v) \nabla v=\frac{1}{\sqrt{a(g)}} \nabla v
$$

hence

$$
\nabla v=\sqrt{a(u)} \nabla u
$$

which leads to

$$
\Delta v=\sqrt{a(u)} \Delta u+\frac{1}{2} \frac{1}{\sqrt{a(u)}} a^{\prime}(u)|\nabla u|^{2} .
$$

Hence (2.2) implies

$$
\sqrt{a(u)} \Delta u+\frac{1}{2} \frac{1}{\sqrt{a(u)}} a^{\prime}(u)|\nabla u|^{2}=\frac{f(u)}{\sqrt{a(u)}}
$$


which is equivalent to (2.1). Hence, (2.1) admits a point rupture solution if and only if (2.2) has a point rupture solution.

Noticing that $h(v)=\frac{f(g(v))}{\sqrt{a(g(v))}}$ is not necessary monotone decreasing in $v$. However, the boundedness of $a$ and the monotone properties of $f$ and $g$ implies that

$$
\frac{1}{\sqrt{M}} f(g(v)) \leq h(v) \leq \frac{1}{\sqrt{m}} f(g(v)) \text { for any } v \in\left[0, g^{-1}\left(\delta^{*}\right)\right],
$$

i.e., $h$ is bounded by two monotone decreasing functions.

We have the following existence result on rupture solutions to (2.2):

Proposition 1. Let $\sigma^{*}>0$ and $h_{1}, h_{2} \in C^{1}\left(0, \sigma^{*}\right]$ be monotone decreasing functions such that

$$
0<h_{1} \leq h_{2} \text { on }\left(0, \sigma^{*}\right]
$$

and

$$
\lim _{v \rightarrow 0^{+}} h_{1}(v)=\lim _{v \rightarrow 0^{+}} h_{2}(v)=\infty
$$

Let $h \in C^{1}\left(0, \sigma^{*}\right]$ satisfy

$$
h_{1} \leq h \leq h_{2} \text { on }\left(0, \sigma^{*}\right] .
$$

Let

$$
G_{1}(v)=\int_{0}^{v} \frac{1}{h_{1}(s)} \mathrm{d} s
$$

Assume in addition that

$$
\frac{h_{2}}{h_{1}} \in L^{1}\left[0, \sigma^{*}\right] \text { and } \frac{\int_{0}^{v} \frac{h_{2}(s)}{h_{1}(s)} \mathrm{d} s}{G_{1}(v) h_{1}(v)} \in L^{1}\left[0, \sigma^{*}\right] .
$$

Then there exists $r^{*}>0$ and a radial point rupture solution $v_{0}$ to

$$
\Delta v=h(v)
$$

in $B_{r^{*}}(0)$ such that $v_{0}=v_{0}(r)$ is continuous on $\left[0, r^{*}\right]$,

$$
v_{0}(0)=0, v_{0}(r)>0 \text { for any } r \in\left(0, r^{*}\right] .
$$

Moreover, $v_{0}$ is monotone increasing and

$$
G_{1}^{-1}\left(\frac{1}{4} r^{2}\right) \leq v_{0}(r) \leq \int_{0}^{G_{1}^{-1}\left(\frac{1}{4} r^{2}\right)} \frac{\int_{0}^{v} \frac{h_{2}(s)}{h_{1}(s)} \mathrm{d} s}{G_{1}(v) h_{1}(v)} \mathrm{d} v \text { for any } r \in\left[0, r^{*}\right] .
$$

For any $\sigma \in\left(0, \sigma^{*}\right)$, we use $v_{\sigma}$ to denote the unique solution to the initial value problem

$$
\left\{\begin{array}{c}
v_{r r}+\frac{1}{r} v_{r}=h(v) \\
v(0)=\sigma, v^{\prime}(0)=0 .
\end{array}\right.
$$

Lemma 1. There exists $r_{\sigma}>0$ such that $v_{\sigma}$ is defined on $\left[0, r_{\sigma}\right]$ with $v_{\sigma}\left(r_{\sigma}\right)=\sigma^{*}$. Moreover, $v_{\sigma}^{\prime}(r)>0$ on $\left(0, r_{\sigma}\right]$ and

$$
G^{-1}\left(\frac{1}{4} r^{2}\right) \leq v_{\sigma}(r) \leq \sigma+H\left(G_{1}^{-1}\left(\frac{1}{4} r^{2}\right)\right) \text { on }\left[0, r_{\sigma}\right] \text {. }
$$

where

$$
H(w)=\int_{0}^{w} \frac{\int_{0}^{v} \frac{h_{2}(s)}{h_{1}(s)} \mathrm{d} s}{G_{1}(v) h_{1}(v)} \mathrm{d} v
$$


Proof. For simplicity, we suppress the $\sigma$ subscript in this proof. We write

$$
v_{r r}+\frac{1}{r} v_{r}=h(v)
$$

in the form of

$$
\left(r v_{r}\right)_{r}=r h(v) \geq 0
$$

so we have

$$
r v_{r}=\int_{0}^{r} \operatorname{sh}(v(s)) \mathrm{d} s \geq 0 .
$$

In particular, $v$ is monotone increasing and $v$ can be extended whenever $h(v)$ is defined and bounded. Hence, there exists $r_{\sigma}>0$ such that $v_{\sigma}$ is defined on $\left[0, r_{\sigma}\right]$ with $v_{\sigma}\left(r_{\sigma}\right)=\sigma^{*}$. Since $v$ is monotone increasing and $h_{1}$ is monotone decreasing, we have

$$
\begin{aligned}
r v_{r} & =\int_{0}^{r} s h(v(s)) \mathrm{d} s \geq \int_{0}^{r} s h_{1}(v(s)) \mathrm{d} s \\
& \geq h_{1}(v(r)) \int_{0}^{r} s \mathrm{~d} s=\frac{1}{2} r^{2} h_{1}(v(r)),
\end{aligned}
$$

hence,

$$
\frac{v_{r}}{h_{1}(v)} \geq \frac{1}{2} r
$$

Integrating again, we have

$$
G_{1}(v(r)) \geq G_{1}(\sigma)+\frac{1}{4} r^{2} \geq \frac{1}{4} r^{2}
$$

where

$$
G_{1}(v)=\int_{0}^{v} \frac{1}{h_{1}(s)} \mathrm{d} s
$$

Since $G_{1}$ is continuous and strictly monotone increasing, $G_{1}^{-1}$ is well defined and we have

$$
v(r) \geq G_{1}^{-1}\left(\frac{1}{4} r^{2}\right)
$$

On the other hand, since $h_{2}$ is monotone increasing,

$$
r v_{r}=\int_{0}^{r} s h(v(s)) \mathrm{d} s \leq \int_{0}^{r} s h_{2}(v(s)) \mathrm{d} s \leq \int_{0}^{r} h_{2}\left(G_{1}^{-1}\left(\frac{1}{4} s^{2}\right)\right) s \mathrm{~d} s .
$$

Let $v=G_{1}^{-1}\left(\frac{1}{4} s^{2}\right)$, we have $G_{1}(v)=\frac{1}{4} s^{2}$, and

$$
\frac{1}{h_{1}(v)} \mathrm{d} v=\frac{1}{2} s \mathrm{~d} s
$$

Hence,

$$
\int_{0}^{r} h_{2}\left(G_{1}^{-1}\left(\frac{1}{4} s^{2}\right)\right) s \mathrm{~d} s=2 \int_{0}^{G_{1}^{-1}\left(\frac{1}{4} r^{2}\right)} \frac{h_{2}(v)}{h_{1}(v)} \mathrm{d} v
$$

Hence,

$$
v_{r} \leq \frac{2}{r} \int_{0}^{G_{1}^{-1}\left(\frac{1}{4} r^{2}\right)} \frac{h_{2}(s)}{h_{1}(s)} \mathrm{d} s
$$


which yields

$$
\begin{aligned}
v(r) & \leq \sigma+\int_{0}^{r} \frac{2}{s}\left[\int_{0}^{G_{1}^{-1}\left(\frac{1}{4} s^{2}\right)} \frac{h_{2}(t)}{h_{1}(t)} \mathrm{d} t\right] \mathrm{d} s \\
& =\sigma+\int_{0}^{G_{1}^{-1}\left(\frac{1}{4} r^{2}\right)} \frac{2}{s}\left[\int_{0}^{w} \frac{h_{2}(t)}{h_{1}(t)} \mathrm{d} t\right] \frac{2}{s h_{1}(w)} d w \\
& =\sigma+\int_{0}^{G_{1}^{-1}\left(\frac{1}{4} r^{2}\right)} \frac{\int_{0}^{w} \frac{h_{2}(t)}{h_{1}(t)} \mathrm{d} t}{G_{1}(w) h_{1}(w)} d w \\
& =\sigma+H\left(G_{1}^{-1}\left(\frac{1}{4} r^{2}\right)\right)
\end{aligned}
$$

where

$$
H(w)=\int_{0}^{w} \frac{\int_{0}^{s} \frac{h_{2}(t)}{h_{1}(t)} \mathrm{d} t}{G_{1}(s) h_{1}(s)} \mathrm{d} s
$$

and we used substitution

$$
w=G_{1}^{-1}\left(\frac{1}{4} s^{2}\right) .
$$

The bounds on $v_{\sigma}$ imply:

Corollary 1. There exists $r^{*}>0$ such that for any $\sigma \in\left(0, \frac{\sigma^{*}}{2}\right]$,

$$
r_{\sigma} \geq r^{*}
$$

We can take

$$
r^{*}=2 \sqrt{G_{1}\left(H^{-1}\left(\frac{\sigma^{*}}{2}\right)\right)} .
$$

Proof. For any $\sigma \in\left(0, \frac{\sigma^{*}}{2}\right]$,

$$
\begin{aligned}
\sigma^{*} & =v_{\sigma}\left(r_{\sigma}\right) \leq \sigma+H\left(G_{1}^{-1}\left(\frac{1}{4} r_{\sigma}^{2}\right)\right) \\
& \leq \frac{\sigma^{*}}{2}+H\left(G_{1}^{-1}\left(\frac{1}{4} r_{\sigma}^{2}\right)\right) .
\end{aligned}
$$

Hence,

$$
H\left(G_{1}^{-1}\left(\frac{1}{4} r_{\sigma}^{2}\right)\right) \geq \frac{\sigma^{*}}{2} .
$$

Since the function $H$ is strictly monotone increasing, we have

$$
G_{1}^{-1}\left(\frac{1}{4} r_{\sigma}^{2}\right) \geq H^{-1}\left(\frac{\sigma^{*}}{2}\right)
$$

and since $G_{1}$ is strictly monotone increasing, we have

$$
r_{\sigma} \geq 2 \sqrt{G_{1}\left(H^{-1}\left(\frac{\sigma^{*}}{2}\right)\right)} .
$$

The point rupture solution can be constructed as the limit of $v_{\sigma}$ as $\sigma \rightarrow 0$. 
Proof of Proposition 1. For any $\varepsilon>0, v_{\sigma}, \sigma \in\left(0, \frac{\sigma^{*}}{2}\right]$ is a family of uniformly bounded classical solutions to

$$
\Delta v=h(v) \text { in } \overline{B_{r^{*}}(0)} \backslash B_{\varepsilon}(0),
$$

hence by a diagonal argument, there exists a sequence $\left\{\sigma_{k}\right\}_{k=1}^{\infty} \subset\left(0, \frac{\sigma^{*}}{2}\right]$ satisfying $\lim _{k \rightarrow \infty} \sigma_{k}=0$, such that $v_{\sigma_{k}} \rightarrow v_{0}$ locally uniformly in $\overline{B_{r^{*}}(0)} \backslash\{0\}$ as $k \rightarrow \infty$. Now (2.7) implies

$$
G_{1}^{-1}\left(\frac{1}{4} r^{2}\right) \leq v_{0}(r) \leq H\left(G_{1}^{-1}\left(\frac{1}{4} r^{2}\right)\right) \text { on }\left[0, r^{*}\right]
$$

Since

$$
\lim _{r \rightarrow 0} H\left(G_{1}^{-1}\left(\frac{1}{4} r^{2}\right)\right)=0
$$

it is not difficulty to see, from the bounds of $v_{\sigma}$ and $v_{0}$, that $v_{\sigma_{k}} \rightarrow v_{0}$ uniformly in $\overline{B_{r^{*}}(0)}$ as $k \rightarrow \infty$. The above bounds also imply that $v_{0}(0)=0$ and $v_{0}(r)>0$ for any $r \in\left(0, r^{*}\right]$. Standard elliptic theory implies that $v_{0} \in C^{2, \alpha}\left(B_{r^{*}}(0) \backslash\{0\}\right)$ and

$$
\Delta v_{0}=h\left(v_{0}\right) \text { in } B_{r^{*}}(0) \backslash\{0\} .
$$

Hence $v_{0}$ is a rupture solution.

Remark 1. The above limit in the proof should be independent of the choice of $\left\{\sigma_{k}\right\}_{k=1}^{\infty}$. Actually, we expect that $v_{\sigma} \rightarrow v_{0}$ uniformly on $\left[0, r^{*}\right]$ as $\sigma \rightarrow 0$. Unfortunately, we are unable to provide a proof here.

Even though $v_{0}$ is continuous, its derivatives have singularity at the origin. Now we investigate the behavior of $v_{0}$ near the origin:

Lemma 2. The rupture solution $v_{0} \in H_{l o c}^{1}\left(B_{r^{*}}(0)\right)$ and $f\left(v_{0}\right) \in H_{l o c}^{1}\left(B_{r^{*}}(0)\right)$ and

$$
\lim _{r \rightarrow 0^{+}} r v_{0}^{\prime}(r)=0 \text {. }
$$

Proof. For any $r \in\left(0, r^{*}\right)$, we have

$$
\left(r v_{0}^{\prime}(r)\right)^{\prime}=r f\left(v_{0}\right)>0 .
$$

Hence, $r v_{0}^{\prime}(r)$ is monotone increasing in $\left(0, r^{*}\right)$. Since $r v_{0}^{\prime}(r) \geq 0$ in $\left(0, r^{*}\right)$,

$$
\beta=\lim _{r \rightarrow 0^{+}} r v_{0}^{\prime}(r) \geq 0
$$

is well defined. If $\beta>0$, we have for $r$ sufficiently small, say $r \in(0, \tilde{r}]$,

$$
r v_{0}^{\prime}(r) \geq \frac{\beta}{2}
$$

hence, for any $r \in(0, \tilde{r}]$,

$$
v_{0}(r)=v_{0}(\tilde{r})-\int_{r}^{\tilde{r}} v_{0}^{\prime}(r) \mathrm{d} r \leq v_{0}(\tilde{r})-\int_{r}^{\tilde{r}} \frac{\beta}{2 r} \mathrm{~d} r .
$$

which contradicts to the fact that $v_{0}$ is continuous at 0 if we let $r \rightarrow 0^{+}$. Hence $\beta=0$ and $(2.8)$ holds.

Next, for any $\varepsilon \in\left(0, r^{*} / 2\right)$,

$$
\begin{aligned}
& \int_{B_{r^{*} / 2}(0) \backslash \overline{B_{\varepsilon}(0)}} h\left(v_{0}\right) d x \\
& =\int_{B_{r^{*} / 2}(0) \backslash \frac{B_{\varepsilon}(0)}{\partial v_{0}}} \Delta v_{0} d x \\
& =\int_{\partial B_{r^{*} / 2}(0)} \frac{\partial s_{x}-\int_{\partial B_{\varepsilon}(0)} \frac{\partial v_{0}}{\partial r} \mathrm{~d} s_{x} .}{}
\end{aligned}
$$


Since

we have

$$
\lim _{\varepsilon \rightarrow 0^{+}}\left|\int_{\partial B_{\varepsilon}(0)} \frac{\partial v_{0}}{\partial r} \mathrm{~d} s_{x}\right|=\lim _{\varepsilon \rightarrow 0} 2 \pi \varepsilon v_{0}^{\prime}(\varepsilon)=0,
$$

$$
\lim _{\varepsilon \rightarrow 0^{+}} \int_{B_{r^{*} / 2}(0) \backslash \overline{B_{\varepsilon}(0)}} h\left(v_{0}\right) d x=\int_{\partial B_{r^{*} / 2}(0)} \frac{\partial v_{0}}{\partial r} \mathrm{~d} s_{x}
$$

hence, $h\left(v_{0}\right) \in L_{l o c}^{1}\left(B_{r^{*}}(0)\right)$. Similarly, for any $\varepsilon \in\left(0, r^{*} / 2\right)$,

$$
\begin{aligned}
& \int_{B_{r^{*} / 2}(0) \backslash \overline{B_{\varepsilon}(0)}}\left|\nabla v_{0}\right|^{2} d x \\
& =-\int_{B_{r^{*} / 2}(0) \backslash \overline{B_{\varepsilon}(0)}} v_{0} \Delta v_{0} d x+\int_{\partial B_{r^{*} / 2}(0)} v_{0} v_{0}^{\prime} \mathrm{d} s_{x}-\int_{\partial B_{\varepsilon}(0)} v_{0} v_{0}^{\prime} \mathrm{d} s_{x} \\
& =-\int_{B_{r^{*} / 2}(0) \backslash \overline{B_{\varepsilon}(0)}} v_{0} h\left(v_{0}\right) d x+\int_{\partial B_{r^{*} / 2}(0)} v_{0} v_{0}^{\prime} \mathrm{d} s_{x}-\int_{\partial B_{\varepsilon}(0)} v_{0} v_{0}^{\prime} \mathrm{d} s_{x},
\end{aligned}
$$

Letting $\varepsilon \rightarrow 0$, we have

$$
\lim _{\varepsilon \rightarrow 0^{+}} \int_{B_{r^{*} / 2}(0) \backslash \overline{B_{\varepsilon}(0)}}\left|\nabla v_{0}\right|^{2} d x=-\int_{B_{r^{*} / 2}(0)} v_{0} h\left(v_{0}\right) d x+\int_{\partial B_{r^{*} / 2}(0)} v_{0} v_{0}^{\prime} \mathrm{d} s_{x},
$$

hence $\left|\nabla v_{0}\right|^{2} \in L_{l o c}^{1}\left(B_{r^{*}}(0)\right)$ and $v_{0} \in H_{l o c}^{1}\left(B_{r^{*}}(0)\right)$.

Now we are ready to prove our main theorem:

Proof of Theorem 1. Let $\sigma^{*}=g^{-1}\left(\delta^{*}\right)$, and for any $v \in\left(0, \sigma^{*}\right]$, define

$$
h_{1}(v)=\frac{1}{\sqrt{M}} f(g(v)) \text { and } h_{2}(v)=\frac{1}{\sqrt{m}} f(g(v)) .
$$

We have

$$
h_{1}(v) \leq h(v)=\frac{f(g(v))}{\sqrt{a(g(v))}} \leq h_{2}(v)
$$

on $\left(0, \sigma^{*}\right]$. It is easy to verify that the assumption on 1 holds for $h$. In particular, we have

$$
\frac{h_{2}}{h_{1}}=\frac{\sqrt{M}}{\sqrt{m}} \in L^{1}\left[0, \sigma^{*}\right],
$$

and for any $v \in\left(0, \sigma^{*}\right]$,

$$
\begin{aligned}
G_{1}(v) & =\int_{0}^{v} \frac{1}{h_{1}(s)} \mathrm{d} s .=\sqrt{M} \int_{0}^{v} \frac{1}{f(g(s))} \mathrm{d} s \\
& =\sqrt{M} \int_{0}^{v} \frac{\sqrt{a(g)}}{f(g(s))} g^{\prime}(s) \mathrm{d} s \\
& =\sqrt{M} \int_{0}^{g(v)} \frac{\sqrt{a(u)}}{f(u)} d u \\
& \geq \sqrt{m M} \int_{0}^{g(v)} \frac{1}{f(u)} d u=\sqrt{m M} G(g(v)),
\end{aligned}
$$

and

$$
\begin{aligned}
\frac{\int_{0}^{v} \frac{h_{2}(s)}{h_{1}(s)} \mathrm{d} s}{G_{1}(v) h_{1}(v)} & =\frac{\sqrt{M}}{\sqrt{m}} \frac{u}{G_{1}(v) \frac{1}{\sqrt{M}} f(g(v))} \\
& \leq \frac{\sqrt{M}}{m} \frac{v}{G(g(v)) f(g(v))} \\
& \leq \frac{M}{m} \frac{g(v)}{G(g(v)) f(g(v))}
\end{aligned}
$$


where we used

$$
g(v) \geq \frac{1}{\sqrt{M}} v
$$

Hence,

$$
\begin{aligned}
& \int_{0}^{\sigma^{*}} \frac{\int_{0}^{v} \frac{h_{2}(s)}{h_{1}(s)} \mathrm{d} s}{G_{1}(v) h_{1}(v)} \mathrm{d} v \\
& \leq \int_{0}^{\sigma^{*}} \frac{M}{m} \frac{g(v)}{G(g(v)) f(g(v))} \mathrm{d} v \\
& =\int_{0}^{\sigma^{*}} \frac{M}{m} \frac{g(v)}{G(g(v)) f(g(v))} \sqrt{a(g)} g^{\prime}(v) \mathrm{d} v \\
& \leq \frac{M \sqrt{M}}{m} \int_{0}^{\delta^{*}} \frac{u}{G(u) f(u)} d u .
\end{aligned}
$$

So the growth condition in (1.3) implies that,

$$
\frac{\int_{0}^{u} \frac{h_{2}(v)}{h_{1}(v)} \mathrm{d} v}{G_{1}(u) h_{1}(u)} \in L^{1}\left[0, \sigma^{*}\right] .
$$

Proposition 1 implies the existence of a rupture solution $v_{0}$ to $(2.5)$, hence

$$
u_{0}=g\left(v_{0}\right)
$$

is a rupture solution to (1.2). The properties for $v_{0}$ imply that $u_{0} \in H_{l o c}^{1}\left(B_{1}\left(r^{*}\right)\right), f\left(u_{0}\right) \in L_{l o c}^{1}\left(B_{1}\left(r^{*}\right)\right)$ and

$$
\lim _{r \rightarrow 0^{+}} r u_{0}^{\prime}(r)=0
$$

For any any $\varphi \in C_{c}^{\infty}\left(B_{r^{*}}(0)\right)$, we have

$$
\begin{aligned}
& \int_{B_{r^{*}}(0)} a\left(u_{0}\right) \nabla u_{0} \nabla \varphi d x=\lim _{\varepsilon \rightarrow 0^{+}} \int_{B_{r^{*}}(0) \backslash \overline{B_{\varepsilon}(0)}} a\left(u_{0}\right) \nabla u_{0} \nabla \varphi d x \\
= & \lim _{\varepsilon \rightarrow 0^{+}}\left(-\int_{B_{r^{*}}(0) \backslash \overline{B_{\varepsilon}(0)}} \operatorname{div}\left(a\left(u_{0}\right) \nabla u_{0}\right) \varphi d x-\int_{\partial B_{\varepsilon}(0)}\left(a\left(u_{0}\right) \frac{\partial u_{0}}{\partial r} \varphi\right) \mathrm{d} s_{x}\right) \\
= & \lim _{\varepsilon \rightarrow 0^{+}}\left(\int_{B_{r^{*}}(0) \backslash \overline{B_{\varepsilon}(0)}}\left(\frac{a^{\prime}\left(u_{0}\right)}{2}\left|\nabla u_{0}\right|^{2}+f\left(u_{0}\right)\right) \varphi d x-\int_{\partial B_{\varepsilon}(0)}\left(a\left(u_{0}\right) \frac{\partial u_{0}}{\partial r} \varphi\right) \mathrm{d} s_{x}\right) \\
= & \int_{B_{r^{*}}(0)}\left(\frac{a^{\prime}\left(u_{0}\right)}{2}\left|\nabla u_{0}\right|^{2}+f\left(u_{0}\right)\right) \varphi d x .
\end{aligned}
$$

Hence $u_{0}$ is a weak solution to (1.2) in $B_{r^{*}}(0)$.

We discuss several examples at the end of this section to get a better understanding of the technical assumption on the growth rate of $h$ in (2.4).

\section{Example 1.}

$$
h(v)=b(v) v^{-\alpha}
$$

where $\alpha>0$ is a constant and $b(v)$ satisfies

$$
B_{1} \leq b(v) \leq B_{2}
$$

for some constants $0<B_{1}<B_{2}$. If we take

$$
h_{1}=B_{1} v^{-\alpha} \text { and } h_{2}=B_{2} v^{-\alpha},
$$

we have

$$
\frac{\int_{0}^{v} \frac{h_{2}(s)}{h_{1}(s)} \mathrm{d} s}{G_{1}(v) h_{1}(v)}=\frac{(1+\alpha) B_{2}}{B_{1}} \in L^{1}[0,1] .
$$


Example 2. For some $0<p<1$,

$$
h(v)=b(v) v^{p+1} e^{\frac{1}{v^{p}}}
$$

and $b(u)$ satisfies

$$
B_{1} \leq b(v) \leq B_{2}
$$

for some constants $0<B_{1}<B_{2}$. If we take

$$
h_{1}=B_{1} v^{p+1} e^{\frac{1}{v^{p}}} \text { and } h_{2}=B_{2} v^{p+1} e^{\frac{1}{v^{p}}},
$$

we have

\section{Example 3.}

$$
\frac{\int_{0}^{v} \frac{h_{2}(s)}{h_{1}(s)} \mathrm{d} s}{G_{1}(v) h_{1}(v)}=\frac{B_{2} p}{B_{1} v^{p}} \in L^{1}[0,1] .
$$

$$
f(v)=\frac{1}{2}\left[\left(1+\sin \frac{1}{v}\right) v^{-\alpha}+\left(1-\sin \frac{1}{v}\right) v^{-\beta}\right]
$$

where

$$
0<\alpha<\beta<\alpha+1
$$

We take

$$
f_{1}(v)=v^{-\alpha}, f_{2}(v)=v^{-\beta},
$$

then we have for any $v \in(0,1]$,

$$
h_{1}(v) \leq h(v) \leq h_{2}(v) .
$$

Hence,

$$
\frac{\int_{0}^{v} \frac{h_{2}(s)}{h_{1}(s)} \mathrm{d} s}{G_{1}(v) h_{1}(v)}=\frac{\int_{0}^{v} s^{\alpha-\beta} \mathrm{d} s}{\frac{1}{1+\alpha} v}=\frac{1+\alpha}{1+\alpha-\beta} v^{\alpha-\beta} \in L^{1}[0,1]
$$

since $\alpha-\beta>-1$. In this example, $h$ can't be expressed as a product of a bounded function and a monotone function.

Example 4. This example shows that our result is not optimal. Let

$$
h(v)=2 v^{3} e^{\frac{2}{v}}
$$

which is monotone decreasing near the origin and

$$
\lim _{v \rightarrow 0^{+}} h(v)=\infty
$$

Taking

$$
h_{1}(v)=h_{2}(v)=h(v),
$$

we have

$$
\begin{aligned}
\frac{\int_{0}^{v} \frac{h_{2}(s)}{h_{1}(s)} \mathrm{d} s}{G_{1}(v) h_{1}(v)} & =\frac{v}{2 v^{3} e^{\frac{2}{v}} \int_{0}^{v} \frac{1}{2 s^{3}} e^{-\frac{2}{s}} \mathrm{~d} s} \\
& =\frac{e^{-\frac{2}{v}}}{v^{2} \int_{0}^{v} \frac{1}{s^{3}} e^{-\frac{2}{s}} \mathrm{~d} s}=\frac{e^{-\frac{2}{v}}}{\frac{1}{4}\left(v^{2}+2 v\right) e^{-\frac{2}{v}}} \\
& =\frac{4}{v(2+v)} \notin L^{1}(0, \sigma]
\end{aligned}
$$

for any $\sigma>0$. However, let

$$
v=\frac{-1}{\ln r}
$$

we have

and so

$$
v_{r}=\frac{1}{r \ln ^{2} r}, v_{r r}=-\frac{1}{r^{2} \ln ^{2} r}-2 \frac{1}{r^{2} \ln ^{3} r},
$$

$$
u_{r r}+\frac{1}{r} u_{r}=-2 \frac{1}{r^{2} \ln ^{3} r}=2 v^{3} e^{\frac{2}{v}}=h(v)
$$

Hence $v=\frac{-1}{\ln r}$ is a rupture solution to $\Delta v=h(v)$ even if the technical assumption is not satisfied. 


\section{References}

1. Francesca Gladiali and Marco Squassina. On Explosive Solutions for a Class of Quasi-linear Elliptic Equations. Advanced Nonlinear Studies, 13:663-698, 2013.

2. Zongming Guo, Dong Ye, and Feng Zhou. Existence of singular positive solutions for some semilinear elliptic equations. Pacific J. Math., 236(1):57-71, 2008.

3. Huiqiang Jiang. Energy minimizers of a thin film equation with Born repulsion force. Commun. Pure Appl. Anal., 10(2):803-815, 2011.

4. Huiqiang Jiang and Fanghua Lin. Zero set of soblev functions with negative power of integrability. Chinese Ann. Math. Ser. B, 25(1):65-72, 2004.

5. Huiqiang Jiang and Attou Miloua. Point rupture solutions of a singular elliptic equation. Electronic Journal of Differential Equations, 2013(70):1-8, 2013.

6. Huiqiang Jiang and Wei-Ming Ni. On steady states of van der Waals force driven thin film equations. European J. Appl. Math., 18(2):153-180, 2007. 